THU-97-44

NIKHEF 97-045

hep-th/9710215

\title{
Open and Closed Supermembranes with Winding
}

\author{
Bernard de Wit ${ }^{\mathrm{a}}$, Kasper Peeters ${ }^{\mathrm{b}}$ and Jan C. Plefka ${ }^{\mathrm{b}}$ \\ anstitute for Theoretical Physics, Utrecht University \\ Princetonplein 5, 3508 TA Utrecht, The Netherlands \\ bNIKHEF, P.O. Box 41882, 1009 DB Amsterdam, The Netherlands
}

\begin{abstract}
Motivated by manifest Lorentz symmetry and a well-defined large- $N$ limit prescription, we study the supersymmetric quantum mechanics proposed as a model for the collective dynamics of D0-branes from the point of view of the 11-dimensional supermembrane. We argue that the continuity of the spectrum persists irrespective of the presence of winding around compact target-space directions and discuss the central charges in the superalgebra arising from winding membrane configurations. Along the way we comment on the structure of open supermembranes.
\end{abstract}

M-theory is defined as the strong-coupling limit of type-IIA string theory and is supposed to capture all the relevant degrees of freedom of all known string theories, both at the perturbative and the nonperturbative level [1,2,3, 31. In this description the various string-string dualities play a central role. At large distances M-theory is described by 11-dimensional supergravity. In [5] it was shown that elementary supermembranes can live in a superspace background that is a solution of the source-free supergravity field equations in 11 dimensions. In the light-cone gauge (in a flat target space) it was subsequently shown [6] that the supermembrane theory takes the form of a supersymmetric quantum-mechanical model, which coincides with the zero-volume reduction of supersymmetric Yang-Mills theory based on $16 \mathrm{su}-$ percharges. For the supermembrane the underlying gauge group is the group of area-preserving diffeomorphisms of the two-dimensional membrane surface. This group can be described by the $N \rightarrow \infty$ limit of SU(N) (the role of the membrane topology is subtle, as we will discuss in due course). For the finite groups the phase-space variables take the form of matrices, associated with the Lie algebra of some group (in this case $\mathrm{U}(N)$ or $\mathrm{SU}(N))$. For this reason, these models are commonly referred to as matrix models. It has been discussed that the possible massless ground states of the supermembrane coincide with the physical states of 11-dimensional supergravityl.

\footnotetext{
${ }^{1}$ For discussions on the existence of massless states, see
}

More recently it was shown that the same quantum-mechanical matrix models based on $\mathrm{U}(N)$ describe the short-distance dynamics of $N$ D0-branes 10,11. Subsequently there has been a large number of studies of these models for finite $N$ 12,13 and some of them have been reported at this conference. These studies were further motivated by a conjecture according to which the degrees of freedom captured in M-theory, are in fact described by the $\mathrm{U}(N)$ super-matrix models in the $N \rightarrow \infty$ limit [14]. A further conjecture, also discussed at this conference, is that the finite- $N$ matrix model coincides with M-theory compactified on a light-like circle [15].

So it turns out that M-theory, supermembranes and super-matrix models are intricately related. A direct relation between supermembranes and type-IIA theory was emphasized in particular in [1], based on the relation between $d=10$ extremal black holes in 10-dimensional supergravity and the Kaluza-Klein states of 11-dimensional supergravity. From the string point of view these states carry Ramond-Ramond charges, just as the D0-branes [16]. Strings can arise from membranes by a so-called double-dimensional reduction [17]. Similarly supermembranes were employed to provide evidence for the duality of M-theory on $\mathbf{R}^{10} \times S_{1} / \mathbf{Z}_{2}$ and 10-dimensional $E_{8} \times E_{8}$ heterotic strings [3].

Here we choose the supermembrane perspec-

6, 8. 9cording to 9 such states do indeed exist in eleven dimensions. 
tive, motivated by its manifest Lorentz invariance and well-defined large- $N$ limit (but not necessarily committing ourselves to the view that Mtheory is a theory of fundamental membranes). Let us nevertheless first consider the supersymmetric matrix models, whose Hamiltonian equals

$H=\frac{1}{g} \operatorname{Tr}\left[\frac{1}{2} \mathbf{P}^{2}+\frac{1}{4}\left[X^{a}, X^{b}\right]^{2}+g \theta^{\mathrm{T}} \gamma_{a}\left[X^{a}, \theta\right]\right]$

Here, $\mathbf{X}, \mathbf{P}$ and $\theta$ take values in the Lie algebra of the gauge group. From the supermembrane point of view they are vectors and spinors of the 'transverse' $\mathrm{SO}(9)$ rotation group. This Hamiltonian can alternatively be interpreted as the zerovolume limit of supersymmetric Yang-Mills theory with some arbitrary gauge group. For the supermembrane one must choose the (infinitedimensional) group of area-preserving diffeomorphisms (which also plays an important role in selfdual gravity and $N=2$ strings) and put $g$ equal to the light-cone momentum $P_{0}^{+}$. The matrix model has 16 supercharges, but additional charges can be obtained by splitting off an abelian factor of the $\mathrm{U}(N)$ gauge group,

$Q^{+}=\operatorname{Tr}\left[\left(2 P^{a} \gamma_{a}+\left[X^{a}, X^{b}\right] \gamma_{a b}\right) \theta\right]$,

$Q^{-}=g \operatorname{Tr}[\theta]$.

For the supermembrane the second charge is associated with the center-of-mass superalgebra (we return to the membrane supersymmetry algebra shortly).

The form of the area-preserving diffeomorphisms that remain as an invariance of the model, depends in general on the topology of the membrane surface. For spherical and toroidal topologies, it has been shown that the algebra can be approximated by $\mathrm{SU}(N)$ in the large- $N$ limit. This limit is subtle. However, once one assumes an infinitesimal gauge group, one can describe a large variety of different theories. For instance, the gauge group $[\mathrm{U}(N)]^{M}$, which is a subgroup of $\mathrm{U}(N \cdot M)$, leads in the limit $M \rightarrow \infty$ to the possibility of describing the collective dynamics of D0branes on a circle by supersymmetric Yang-Mills theories in $1+1$ dimensions 18]. Hence, it is possible to extract extra dimensions from a suitable infinite-dimensional gauge group. Obviously this can be generalized to a hypertorus. Therefore models based on (subgroups of) $\mathrm{U}(\infty)$ can describe an enormous variety of models.

Using the $\mathrm{SU}(N)$ regularisation it was shown in 19 that the spectrum of the supermembrane is continuous, with no mass gap. This result is expected when the supermembrane Hamiltonian is viewed as the Hamiltonian for the collective dynamics of arbitrarily large numbers of D-particles. From the supermembrane point of view, these instabilities arise because arbitrarily long, string-like (zero-area) configurations can form. There are thus no asymptotic membrane states, but rather multimembrane configurations connected by these infinitely thin strings. The massless ground states, which correspond to the states of 11-dimensional supergravity, thus appear in a continuum of multimembrane states.

The connection with M-theory and finite- $\mathrm{N}$ matrix models is made by compactification of the 11-dimensional action on a circle or higherdimensional tori. However, this compactification has only recently been studied 20 from the point of view of the supermembrane. In this talk we discuss the supermembrane with winding, paying attention to the extension of the supersymmetric gauge theory, the Lorentz invariance and the effect of winding on the mass spectrum. Along the way we shall simultaneously develop the theory of open supermembranes, which has recently received some attention [21,22,23].

The actions of fundamental supermembranes are of the Green-Schwarz type [5]. In flat target space, they read

$$
\begin{aligned}
& \mathcal{L}= \sqrt{-g(X, \theta)} \\
&-\epsilon^{i j k}\left[\frac{1}{2} \partial_{i} X^{\mu}\left(\partial_{j} X^{\nu}+\bar{\theta} \gamma^{\nu} \partial_{j} \theta\right)\right. \\
&\left.\quad+\frac{1}{6} \bar{\theta} \gamma^{\mu} \partial_{i} \theta \bar{\theta} \gamma^{\nu} \partial_{j} \theta\right] \bar{\theta} \gamma_{\mu \nu} \partial_{k} \theta,
\end{aligned}
$$

where $X^{\mu}$ denote the 11-dimensional target-space embedding coordinates lying in $T^{d} \times \mathbf{R}^{1,10-d}$ and thus permitting us to have winding on the $d$ dimensional torus $T^{d}$. Moreover we have the fermionic variables $\theta$, which are 32-component Majorana spinors and $g=\operatorname{det} g_{i j}$ with the induced metric

$g_{i j}=\left(\partial_{i} X^{\mu}+\bar{\theta} \gamma^{\mu} \partial_{i} \theta\right)\left(\partial_{j} X^{\nu}+\bar{\theta} \gamma^{\nu} \partial_{j} \theta\right) \eta_{\mu \nu}$. 
Next to supersymmetry the action (3) exhibits an additional local fermionic symmetry called $\kappa$ symmetry. In the case of the open supermembrane, $\kappa$-symmetry imposes boundary conditions on the fields. They must ensure that the following integral over the boundary of the membrane world volume vanishes,

$$
\begin{aligned}
\int_{\partial M}[ & \frac{1}{2} \mathrm{~d} X^{\mu} \wedge\left(\mathrm{d} X^{\nu}+\bar{\theta} \gamma^{\nu} \mathrm{d} \theta\right) \bar{\theta} \gamma_{\mu \nu} \delta_{\kappa} \theta \\
& +\frac{1}{2}\left(\mathrm{~d} X^{\mu}-\frac{1}{3} \bar{\theta} \gamma^{\mu} \mathrm{d} \theta\right) \wedge \bar{\theta} \gamma_{\mu \nu} \mathrm{d} \theta \bar{\theta} \gamma^{\nu} \delta_{\kappa} \theta \\
& \left.+\frac{1}{6} \bar{\theta} \gamma^{\mu} \mathrm{d} \theta \wedge \bar{\theta} \gamma^{\nu} \mathrm{d} \theta \bar{\theta} \gamma_{\mu \nu} \delta_{\kappa} \theta\right] .
\end{aligned}
$$

This can be achieved by having a "membrane D$p$-brane" at the boundary with $p=1,5$, or 9 , which is defined in terms of $(p+1)$ Neumann and $(10-p)$ Dirichlet boundary conditions for the $X^{\mu}$, together with corresponding boundary conditions on the fermionic coordinatest. More explicitly, we define projection operators

$\mathcal{P}_{ \pm}=\frac{1}{2}\left(\mathbf{1} \pm \gamma^{p+1} \gamma^{p+2} \cdots \gamma^{10}\right)$

and impose the Dirichlet boundary conditions

$$
\begin{aligned}
\partial_{\|} X^{M} & =0, \quad M=p+1, \ldots, 10, \\
\mathcal{P}_{-} \theta & =0,
\end{aligned}
$$

where $\partial_{\perp}$ and $\partial_{\|}$define the world-volume derivatives perpendicular or tangential to the surface swept out by the membrane boundary in the target space. Note that the fermionic boundary condition implies that $\mathcal{P}_{-} \partial_{\|} \theta=0$. Furthermore, it implies that spacetime supersymmetry is reduced to only 16 supercharges associated with spinor parameters $\mathcal{P}_{+} \epsilon$, which is chiral with respect to the $(p+1)$-dimensional world volume of the $\mathrm{D}$ $p$-brane at the boundary. With respect to this reduced supersymmetry, the superspace coordinates decompose into two parts, one corresponding to $\left(X^{M}, \mathcal{P}_{-} \theta\right)$ and the other corresponding to $\left(X^{m}, \mathcal{P}_{+} \theta\right)$ where $m=0,1, \ldots, p$. While for the five-brane these superspaces exhibit a somewhat balanced decomposition in terms of an equal number of bosonic and fermionic coordinates, the situation for $p=1,9$ shows heterotic features in

\footnotetext{
${ }^{2}$ Here our conclusions concur with those of 23 but not with those of 22 .
}

that one space has an excess of fermionic and the other an excess of bosonic coordinates. Moreover, we note that supersymmetry may be further broken by e.g. choosing different Dirichlet conditions on non-connected segments of the supermembrane boundary.

The Dirichlet boundary conditions can be supplemented by the following Neumann boundary conditions,

$$
\begin{aligned}
& \partial_{\perp} X^{m} \mid=0 \quad m=0,1, \ldots, p, \\
& \mathcal{P}_{+} \partial_{\perp} \theta \mid=0 .
\end{aligned}
$$

These do not lead to a further breakdown of the rigid spacetime symmetries.

We now continue and follow the light-cone quantization described in [6]. The supermembrane Hamiltonian takes the form

$$
\begin{aligned}
\mathcal{H}=\frac{1}{P_{0}^{+}} \int \mathrm{d}^{2} \sigma \sqrt{w} & {\left[\frac{P^{a} P_{a}}{2 w}+\frac{1}{4}\left\{X^{a}, X^{b}\right\}^{2}\right.} \\
& \left.-P_{0}^{+} \bar{\theta} \gamma_{-} \gamma_{a}\left\{X^{a}, \theta\right\}\right] .
\end{aligned}
$$

Here the integral runs over the spatial components of the worldvolume denoted by $\sigma^{1}$ and $\sigma^{2}$, while $P^{a}(\sigma)(a=2, \ldots, 10)$ are the momentum conjugates to the transverse $X^{a}$. In this gauge the light-cone coordinate $X^{+}=\left(X^{1}+X^{0}\right) / \sqrt{2}$ is linearly related to the world-volume time. The momentum $P^{+}$is time independent and proportional to the center-of-mass value $P_{0}^{+}$times some density $\sqrt{w(\sigma)}$ of the spacesheet, whose spacesheet integral is normalized to unity. The center-of-mass momentum $P_{0}^{-}$is equal to minus the Hamiltonian (9) subject to the gauge condition $\gamma_{+} \theta=0$. And finally we made use of the Poisson bracket $\{A, B\}$ defined by

$\{A(\sigma), B(\sigma)\}=\frac{1}{\sqrt{w(\sigma)}} \epsilon^{r s} \partial_{r} A(\sigma) \partial_{s} B(\sigma)$.

Note that the coordinate $X^{-}=\left(X^{1}-X^{0}\right) / \sqrt{2}$ itself does not appear in the Hamiltonian (9). It is defined via

$P_{0}^{+} \partial_{r} X^{-}=-\frac{\mathbf{P} \cdot \partial_{r} \mathbf{X}}{\sqrt{w}}-P_{0}^{+} \bar{\theta} \gamma_{-} \partial_{r} \theta$

and implies a number of constraints that will be important in the following. First of all, the righthand side must be closed. If there is no winding in $X^{-}$, it must moreover be exact. 
The equivalence of the large- $N$ limit of $\mathrm{SU}(N)$ quantum mechanics with the closed supermembrane model is based on the residual invariance of the supermembrane action in the light-cone gauge. It is given by the area-preserving diffeomorphisms of the membrane surface. These are defined by transformations of the worldsheet coordinates

$\sigma^{r} \rightarrow \sigma^{r}+\xi^{r}(\sigma)$,

with

$\partial_{r}\left(\sqrt{w(\sigma)} \xi^{r}(\sigma)\right)=0$.

We wish to rewrite this condition in terms of dual spacesheet vectors by

$\sqrt{w(\sigma)} \xi^{r}(\sigma)=\epsilon^{r s} F_{s}(\sigma)$.

In the language of differential forms the condition (12) may then be simply recast as $\mathrm{d} F=0$. The trivial solutions are the exact forms $F=\mathrm{d} \xi$, or in components

$F_{s}=\partial_{s} \xi(\sigma)$,

for any globally defined function $\xi(\sigma)$. The nontrivial solutions are the closed forms which are not exact. On a Riemann surface of genus $g$ there are precisely $2 g$ linearly independent non-exact closed forms, whose integrals along the homology cycles are normalized to unity 3 . In components we write

$F_{s}=\phi_{(\lambda) s}, \quad \lambda=1, \ldots, 2 g$.

The commutator of two infinitesimal areapreserving diffeomorphisms is determined by the product rule

$\xi_{r}^{(3)}=\partial_{r}\left(\frac{\epsilon^{s t}}{\sqrt{w}} \xi_{s}^{(2)} \xi_{t}^{(1)}\right)$,

where both $\xi_{r}^{(1,2)}$ are closed vectors. Because $\xi_{r}^{(3)}$ is exact, the exact vectors generate an invariant subgroup of the area-preserving diffeomorphisms, which can be approximated by $\mathrm{SU}(N)$ in the large- $N$ limit in the case of closed membranes.

${ }^{3}$ In the mathematical literature the globally defined exact forms are called "hamiltonian vector fields", whereas the closed but not exact forms which are not globally defined go under the name "locally hamiltonian vector fields".
For open membranes the boundary conditions on the fields (7) lead to an $\mathrm{SO}(N)$ group structure, as we shall see in the sequel.

The presence of the closed but non-exact forms is crucial for the winding of the embedding coordinates. More precisely, while the momenta $\mathbf{P}(\sigma)$ and the fermionic coordinates $\theta(\sigma)$ remain single valued on the spacesheet, the embedding coordinates, written as one-forms with components $\partial_{r} \mathbf{X}(\sigma)$ and $\partial_{r} X^{-}(\sigma)$, are decomposed into closed forms. Their non-exact contributions are multiplied by an integer times the length of the compact direction. The constraint alluded to above amounts to the condition that the right-hand side of (11) is closed.

Under the full group of area-preserving diffeomorphisms the fields $X^{a}, X^{-}$and $\theta$ transform according to

$$
\begin{gathered}
\delta X^{a}=\frac{\epsilon^{r s}}{\sqrt{w}} \xi_{r} \partial_{s} X^{a}, \quad \delta X^{-}=\frac{\epsilon^{r s}}{\sqrt{w}} \xi_{r} \partial_{s} X^{-}, \\
\delta \theta^{a}=\frac{\epsilon^{r s}}{\sqrt{w}} \xi_{r} \partial_{s} \theta
\end{gathered}
$$

where the time-dependent reparametrization $\xi_{r}$ consists of closed exact and non-exact parts. Accordingly there is a gauge field $\omega_{r}$, which is therefore closed as well, transforming as

$\delta \omega_{r}=\partial_{0} \xi_{r}+\partial_{r}\left(\frac{\epsilon^{s t}}{\sqrt{w}} \xi_{s} \omega_{t}\right)$,

and corresponding covariant derivatives

$$
\begin{aligned}
D_{0} X^{a} & =\partial_{0} X^{a}-\frac{\epsilon^{r s}}{\sqrt{w}} \omega_{r} \partial_{s} X^{a}, \\
D_{0} \theta & =\partial_{0} \theta-\frac{\epsilon^{r s}}{\sqrt{w}} \omega_{r} \partial_{s} \theta,
\end{aligned}
$$

and similarly for $D_{0} X^{-}$.

The action corresponding to the following Lagrangian density is then gauge invariant under the transformations (18) and (19),

$$
\begin{aligned}
\mathcal{L}=P_{0}^{+} \sqrt{w}[ & \frac{1}{2}\left(D_{0} \mathbf{X}\right)^{2}+\bar{\theta} \gamma_{-} D_{0} \theta \\
& -\frac{1}{4}\left(P_{0}^{+}\right)^{-2}\left\{X^{a}, X^{b}\right\}^{2} \\
& \left.+\left(P_{0}^{+}\right)^{-1} \bar{\theta} \gamma_{-} \gamma_{a}\left\{X^{a}, \theta\right\}+D_{0} X^{-}\right],
\end{aligned}
$$

where we draw attention to the last term proportional to $X^{-}$, which can be dropped in the ab- 
sence of winding and did not appear in [6]. Moreover, we note that for the open supermembranes, (21) is invariant under the transformations (18) and (19) only if $\xi_{\|}=0$ holds on the boundary. This condition defines a subgroup of the group of area-preserving transformations, which is consistent with the Dirichlet conditions (7). Observe that $\partial_{\|}$and $\partial_{\perp}$ will now refer to the spacesheet derivatives tangential and perpendicular to the membrane boundaryt.

The action corresponding to (21) is also invariant under the supersymmetry transformations

$$
\begin{aligned}
\delta X^{a}= & -2 \bar{\epsilon} \gamma^{a} \theta \\
\delta \theta= & \frac{1}{2} \gamma_{+}\left(D_{0} X^{a} \gamma_{a}+\gamma_{-}\right) \epsilon \\
& \quad+\frac{1}{4}\left(P_{0}^{+}\right)^{-1}\left\{X^{a}, X^{b}\right\} \gamma_{+} \gamma_{a b} \epsilon \\
\delta \omega_{r}= & -2\left(P_{0}^{+}\right)^{-1} \bar{\epsilon} \partial_{r} \theta .
\end{aligned}
$$

The supersymmetry variation of $X^{-}$is not relevant and may be set to zero. For the open case one finds that the boundary conditions $\omega_{\|}=0$ and $\epsilon=\mathcal{P}_{+} \epsilon$ must be fulfilled in order for (22) to be a symmetry of the action. In that case the theory takes the form of a gauge theory coupled to matter. The pure gauge theory is associated with the Dirichlet and the matter with the Neumann (bosonic and fermionic) coordinates.

In the case of a 'membrane D-9-brane' one now sees that the degrees of freedom on the 'end-ofthe world' 9-brane precisely match those of 10dimensional heterotic strings. $O n$ the boundary we are left with eight propagating bosons $X^{m}$ (with $m=2, \ldots, 9$ ), as $X^{10}$ is constant on the boundary due to (7), paired with the 8dimensional chiral spinors $\theta$ (subject to $\gamma_{+} \theta=$ $\left.\mathcal{P}_{-} \theta=0\right)$, i.e., the scenario of Horava-Witten [3].

The full equivalence with the membrane Hamiltonian is now established by choosing the $\omega_{r}=0$ gauge and passing to the Hamiltonian formalism. The field equations for $\omega_{r}$ then lead to the membrane constraint (11) (up to exact contributions), partially defining $X^{-}$. Moreover the Hamiltonian corresponding to the gauge theory Lagrangian of (21) is nothing but the light-cone supermembrane

${ }^{4}$ Consistency of the Neumann boundary conditions (8) with the area-preserving diffeomorphisms (18) further imposes $\partial_{\perp} \xi^{\|}=0$ on the boundary, where indices are raised according to 14 .
Hamiltonian (9). Observe that in the above gauge theoretical construction the space-sheet metric $w_{r s}$ enters only through its density $\sqrt{w}$ and hence vanishing or singular metric components do not pose problems.

We are now in a position to study the full 11dimensional supersymmetry algebra of the winding supermembrane. For this we decompose the supersymmetry charge $Q$ associated with the transformations 22, into two 16-component spinors,

$Q=Q^{+}+Q^{-}, \quad$ where $Q^{ \pm}=\frac{1}{2} \gamma_{ \pm} \gamma_{\mp} Q$,

to obtain

$Q^{+}=\int \mathrm{d}^{2} \sigma\left(2 P^{a} \gamma_{a}+\sqrt{w}\left\{X^{a}, X^{b}\right\} \gamma_{a b}\right) \theta$,

$Q^{-}=2 P_{0}^{+} \int \mathrm{d}^{2} \sigma \sqrt{w} \gamma_{-} \theta$.

The canonical Dirac brackets are derived by the standard methods and read

$$
\begin{aligned}
& \left(X^{a}(\sigma), P^{b}\left(\sigma^{\prime}\right)\right)_{\mathrm{DB}}=\delta^{a b} \delta^{2}\left(\sigma-\sigma^{\prime}\right), \\
& \left(\theta_{\alpha}(\sigma), \bar{\theta}_{\beta}\left(\sigma^{\prime}\right)\right)_{\mathrm{DB}}= \\
& \quad \frac{1}{4}\left(P_{0}^{+}\right)^{-1} w^{-1 / 2}\left(\gamma_{+}\right)_{\alpha \beta} \delta^{2}\left(\sigma-\sigma^{\prime}\right) .
\end{aligned}
$$

In the presence of winding the results given in [6] yield the supersymmetry algebra

$$
\begin{gathered}
\left(Q_{\alpha}^{+}, \bar{Q}_{\beta}^{+}\right)_{\mathrm{DB}}=2\left(\gamma_{+}\right)_{\alpha \beta} \mathcal{H} \\
-2\left(\gamma_{a} \gamma_{+}\right)_{\alpha \beta} \int \mathrm{d}^{2} \sigma \sqrt{w}\left\{X^{a}, X^{-}\right\}, \\
\left(Q_{\alpha}^{+}, \bar{Q}_{\beta}^{-}\right)_{\mathrm{DB}}=-\left(\gamma_{a} \gamma_{+} \gamma_{-}\right)_{\alpha \beta} P_{0}^{a} \\
-\frac{1}{2}\left(\gamma_{a b} \gamma_{+} \gamma_{-}\right)_{\alpha \beta} \int \mathrm{d}^{2} \sigma \sqrt{w}\left\{X^{a}, X^{b}\right\}, \\
\left(Q_{\alpha}^{-}, \bar{Q}_{\beta}^{-}\right)_{\mathrm{DB}}=-2\left(\gamma_{-}\right)_{\alpha \beta} P_{0}^{+},
\end{gathered}
$$

where use has been made of the defining equation (11) for $X^{-}$.

The new feature of this supersymmetry algebra is the emergence of the central charges in the first two anticommutators, which are generated through the winding contributions. They represent topological quantities obtained by integrating the winding densities

$z^{a}(\sigma)=\epsilon^{r s} \partial_{r} X^{a} \partial_{s} X^{-}$ 
and

$z^{a b}(\sigma)=\epsilon^{r s} \partial_{r} X^{a} \partial_{s} X^{b}$

over the space-sheet. It is gratifying to observe the manifest Lorentz invariance of (26). Here we should point out that, in adopting the light-cone gauge, we assumed that there was no winding for the coordinate $X^{+}$. In [28 the corresponding algebra for the matrix regularization was studied. The result obtained in 28] coincides with ours in the large- $N$ limit, in which an additional longitudinal five-brane charge vanishes, provided that one identifies the longitudinal two-brane charge with the central charge in the first line of $(26)$. This requires the definition of $X^{-}$in the matrix regularization, a topic that was dealt with in 24. We observe that the discrepancy noted in 28] between the matrix calculation and certain surface terms derived in [6], seems to have no consequences for the supersymmetry algebra. A possible reason for this could be that certain Schwinger terms have not been treated correctly in the matrix computation, as was claimed in a recent paper 25.

The form of the algebra is another indication of the consistency of the supermembranesupergravity system.

In order to define a matrix approximation one introduces a complete orthonormal basis of functions $Y_{A}(\sigma)$ for the globally defined $\xi(\sigma)$ of $(15)$. One may then write down the following mode expansions for the phase space variables of the supermembrane,

$$
\begin{aligned}
\partial_{r} \mathbf{X}(\sigma) & =\mathbf{X}^{\lambda} \phi_{(\lambda) r}+\sum_{A} \mathbf{X}^{A} \partial_{r} Y_{A}(\sigma), \\
\mathbf{P}(\sigma) & =\sum_{A} \sqrt{w} \mathbf{P}^{A} Y_{a}(\sigma), \\
\theta(\sigma) & =\sum_{A} \theta^{A} Y_{A}(\sigma),
\end{aligned}
$$

introducing winding modes for the transverse $X^{a}$. A similar expansion exists for $X^{-}$. One then naturally introduces the structure constants of the group of area-preserving diffeomorphism by 24.

$$
f_{A B C}=\int \mathrm{d}^{2} \sigma \epsilon^{r s} \partial_{r} Y_{A} \partial_{s} Y_{B} Y_{C},
$$

$$
\begin{aligned}
f_{\lambda B C} & =\int \mathrm{d}^{2} \sigma \epsilon^{r s} \phi_{(\lambda) r} \partial_{s} Y_{B} Y_{C}, \\
f_{\lambda \lambda^{\prime} C} & =\int \mathrm{d}^{2} \sigma \epsilon^{r s} \phi_{(\lambda) r} \phi_{\left(\lambda^{\prime}\right) s} Y_{C} .
\end{aligned}
$$

Note that with $Y_{0}=1$, we have $f_{A B 0}=f_{\lambda B 0}=0$. The raising and lowering of the $A$ indices is performed with the invariant metric

$\eta_{A B}=\int \mathrm{d}^{2} \sigma \sqrt{w} Y_{A}(\sigma) Y_{B}(\sigma)$

and there is no need to introduce a metric for the $\lambda$ indices.

By plugging the mode expansions (29) into the Hamiltonian (9) one obtains the decomposition

$$
\begin{aligned}
\mathcal{H}= & \frac{1}{2} \mathbf{P}_{0} \cdot \mathbf{P}_{0} \\
& +\frac{1}{4} f_{\lambda \lambda^{\prime}}{ }^{0} f_{\lambda^{\prime \prime} \lambda^{\prime \prime \prime} 0} X^{a \lambda} X^{b \lambda^{\prime}} X_{a}^{\lambda^{\prime \prime}} X_{b}^{\lambda^{\prime \prime \prime}} \\
& +\frac{1}{2} \mathbf{P}^{A} \cdot \mathbf{P}_{A}-f_{A B C} \bar{\theta}^{C} \gamma_{-} \gamma_{a} \theta^{B} X^{a A} \\
& -f_{\lambda B C} \bar{\theta}^{C} \gamma_{-} \gamma_{a} \theta^{B} X^{a \lambda} \\
& +\frac{1}{4} f_{A B}{ }^{E} f_{C D E} X^{a A} X^{b B} X_{a}^{C} X_{b}^{D} \\
& +f_{\lambda B}^{E} f_{C D E} X^{a \lambda} X^{b B} X_{a}^{C} X_{b}^{D} \\
& +\frac{1}{2} f_{\lambda B}^{E} f_{\lambda^{\prime} D E} X^{a \lambda} X^{b B} X_{a}^{\lambda^{\prime}} X_{b}^{D} \\
& +\frac{1}{2} f_{\lambda B}{ }^{E} f_{C \lambda^{\prime} E} X^{a \lambda} X^{b B} X_{a}^{C} X_{b}^{\lambda^{\prime}} \\
& +\frac{1}{2} f_{\lambda \lambda^{\prime}}{ }^{E} f_{C D E} X^{a \lambda} X^{b \lambda^{\prime}} X_{a}^{C} X_{b}^{D} \\
& +f_{\lambda \lambda^{\prime}}{ }^{E} f_{\lambda^{\prime \prime} D E} X^{a \lambda} X^{b \lambda^{\prime}} X_{a}^{\lambda^{\prime \prime}} X_{b}^{D} \\
& +\frac{1}{4} f_{\lambda \lambda^{\prime}}{ }^{E} f_{\lambda^{\prime \prime} \lambda^{\prime \prime \prime} E} X^{a \lambda} X^{b \lambda^{\prime}} X_{a}^{\lambda^{\prime \prime}} X_{b}^{\lambda^{\prime \prime \prime}},
\end{aligned}
$$

where here and henceforth we spell out the zeromode dependence explicitly, i.e. the range of values for $A$ no longer includes $A=0$. Note that for the toroidal supermembrane $f_{\lambda \lambda^{\prime} A}=0$ and thus the last three terms in (32) vanish. The second term in the first line represents the winding number squared. In the matrix formulation, the winding number takes the form of a trace over a commutator. We have scaled the Hamiltonian by a factor of $P_{0}^{+}$and the fermionic variables by a factor $\left(P_{0}^{+}\right)^{-1 / 2}$. Supercharges will be rescaled as well, such as to eliminate explicit factors of $P_{0}^{+}$.

The constraint equation (11) is translated into mode language by contracting it with $\epsilon^{r s} \phi_{(\lambda) s}$ and $\epsilon^{r s} \partial_{s} Y_{C}$ respectively and integrating the result over the spacesheet to obtain the two constraints

$\varphi_{\lambda}=f_{\lambda \lambda^{\prime} 0}\left(\mathbf{X}^{\lambda^{\prime}} \cdot \mathbf{P}_{0}+X^{-\lambda^{\prime}} P_{0}^{+}\right)$ 


$$
\begin{gathered}
+f_{\lambda \lambda^{\prime} C} \mathbf{X}^{\lambda^{\prime}} \cdot \mathbf{P}^{C} \\
+f_{\lambda B C}\left(\mathbf{X}^{B} \cdot \mathbf{P}^{C}+\bar{\theta}^{C} \gamma_{-} \theta^{B}\right)=0 \\
\varphi_{A}=f_{A B C}\left(\mathbf{X}^{B} \cdot \mathbf{P}^{C}+\bar{\theta}^{C} \gamma_{-} \theta^{B}\right) \\
+f_{A \lambda C} \mathbf{X}^{\lambda} \cdot \mathbf{P}^{C}=0
\end{gathered}
$$

taking also possible winding in the $X^{-}$direction into account. Note that even for the non-winding case $X^{a \lambda}=0$ there remain the extra $\varphi_{\lambda}$ constraints. These have so far not played any role in the matrix formulation.

The zero-mode contributions completely decouple in the Hamiltonian and the supercharges. We thus perform a split in $Q^{+}$treating zero modes and fluctuations separately to obtain the mode expansions,

$Q^{-}=2 \gamma_{-} \theta^{0}, \quad Q^{+}=Q_{(0)}^{+}+\widehat{Q}^{+}$,

where

$$
\begin{aligned}
Q_{(0)}^{+}= & \left(2 P_{0}^{a} \gamma_{a}+f_{\lambda \lambda^{\prime} 0} X^{a \lambda} X^{b \lambda^{\prime}} \gamma_{a b}\right) \theta_{0}, \\
\widehat{Q}^{+}=( & 2 P_{C}^{a} \gamma_{a}+f_{A B C} X^{a A} X^{b B} \gamma_{a b} \\
& +2 f_{\lambda B C} X^{a \lambda} X^{b B} \gamma_{a b} \\
& \left.+f_{\lambda \lambda^{\prime} C} X^{a \lambda} X^{b \lambda^{\prime}} \gamma_{a b}\right) \theta^{C}
\end{aligned}
$$

Upon introducing the supermembrane mass operator by

$\mathcal{M}^{2}=2 \mathcal{H}-\mathbf{P}_{0} \cdot \mathbf{P}_{0}-\frac{1}{2}\left(f_{\lambda \lambda^{\prime} 0} X^{a \lambda} X^{b \lambda^{\prime}}\right)^{2}$,

the supersymmetry algebra (26) then takes the form

$$
\begin{aligned}
& \left\{\widehat{Q}_{\alpha}^{+}, \bar{Q}_{\beta}^{+}\right\}=\left(\gamma_{+}\right)_{\alpha \beta} \mathcal{M}^{2} \\
& \quad-2\left(\gamma_{a} \gamma_{+}\right)_{\alpha \beta} f_{\lambda \lambda^{\prime} 0} X^{a \lambda}\left(X^{-\lambda^{\prime}} P_{0}^{+}+\mathbf{X}^{\lambda^{\prime}} \cdot \mathbf{P}_{0}\right), \\
& \left\{Q_{(0) \alpha}^{+}, \bar{Q}_{(0) \beta}^{+}\right\}= \\
& \quad\left(\gamma_{+}\right)_{\alpha \beta}\left(\mathbf{P}_{0} \cdot \mathbf{P}_{0}+\frac{1}{2}\left(f_{\lambda \lambda^{\prime} 0} X^{a \lambda} X^{b \lambda^{\prime}}\right)^{2}\right) \\
& \quad+2\left(\gamma_{a} \gamma_{+}\right)_{\alpha \beta} f_{\lambda \lambda^{\prime} 0} X^{a \lambda} \mathbf{X}^{\lambda^{\prime}} \cdot \mathbf{P}_{0}, \\
& \left\{Q_{(0) \alpha}^{+}, \bar{Q}_{\beta}^{-}\right\}=-\left(\gamma_{a} \gamma_{+} \gamma_{-}\right)_{\alpha \beta} P_{0}^{a} \\
& \quad-\frac{1}{2}\left(\gamma_{a b} \gamma_{+} \gamma_{-}\right)_{\alpha \beta} f_{\lambda \lambda^{\prime} 0} X^{a \lambda} X^{b \lambda^{\prime}}, \\
& \left\{\widehat{Q}_{\alpha}^{+}, \bar{Q}_{\beta}^{-}\right\}=\left\{Q_{(0) \alpha}^{+}, \bar{Q}_{\beta}^{+}\right\}=0 .
\end{aligned}
$$

And the mass operator commutes with all the supersymmetry charges,

$\left[\widehat{Q}^{+}, \mathcal{M}^{2}\right]=\left[Q_{(0)}^{+}, \mathcal{M}^{2}\right]=\left[Q^{-}, \mathcal{M}^{2}\right]=0$, defining a supersymmetric quantum-mechanical model.

At this stage it would be desirable to present a matrix model regularization of the supermembrane with winding contributions, generalizing the matrix approximation to the exact subgroup of area-preserving diffeomorphisms [26,6], at least for toroidal geometries. However, this program seems to fail due to the fact that the finite- $N$ approximation to the structure constants $f_{\lambda B C}$ violates the Jacobi identity, as was already noticed in [24].

Let us nevertheless discuss an example of this regularization in the case of non-winding, open supermembranes in some detail. Consider a spacesheet topology of an annulus. Its set of basis functions is easily obtained by starting from the well-known torus functions $Y_{\mathbf{m}}(\sigma)$ [6] labeled by a two-dimensional vector $\mathbf{m}=\left(m_{1}, m_{2}\right)$ with $m_{1}, m_{2}$ integer numbers and

$Y_{\mathbf{m}}\left(\sigma_{1}, \sigma_{2}\right)=e^{i\left(m_{1} \sigma_{1}+m_{2} \sigma_{2}\right)}$.

Consider now the involution $\mathbf{m} \rightarrow \widetilde{\mathbf{m}}$ where $\widetilde{\mathbf{m}}=\left(m_{1},-m_{2}\right)$. One then defines the new basis functions 27,23]

$C_{\mathbf{m}}^{ \pm}=Y_{\mathbf{m}} \pm Y_{\widetilde{\mathbf{m}}}$,

where $\sigma_{1} \in[0,2 \pi]$ and $\sigma_{2} \in[0, \pi]$. It turns out that $C_{\mathbf{m}}^{+}$obeys Neumann and $C_{\mathbf{m}}^{-}$Dirichlet conditions on the boundaries, i.e., $\partial_{2} C_{\mathbf{m}}^{+} \mid=$ $\partial_{1} C_{\mathbf{m}}^{-} \mid=0$. These basis functions possess the algebra

$$
\begin{aligned}
& \left\{C_{\mathbf{m}}^{-}, C_{\mathbf{n}}^{-}\right\}=i(\mathbf{m} \times \mathbf{n}) C_{\mathbf{m}+\mathbf{n}}^{-}-i(\mathbf{m} \times \widetilde{\mathbf{n}}) C_{\mathbf{m}+\widetilde{\mathbf{n}}}^{-}, \\
& \left\{C_{\mathbf{m}}^{+}, C_{\mathbf{n}}^{+}\right\}=i(\mathbf{m} \times \mathbf{n}) C_{\mathbf{m}+\mathbf{n}}^{-}+i(\mathbf{m} \times \widetilde{\mathbf{n}}) C_{\mathbf{m}+\widetilde{\mathbf{n}}}^{-}, \\
& \left\{C_{\mathbf{m}}^{+}, C_{\mathbf{n}}^{-}\right\}=i(\mathbf{m} \times \mathbf{n}) C_{\mathbf{m}+\mathbf{n}}^{+}-i(\mathbf{m} \times \widetilde{\mathbf{n}}) C_{\mathbf{m}+\widetilde{\mathbf{n}}}^{+}
\end{aligned}
$$

Note that the $C_{\mathbf{m}}^{-}$form a closed subalgebra.

The matrix regularization now comes about by replacing $Y_{\mathbf{m}}$ with the $\left(N^{2}-1\right)$ adjoint $\mathrm{SU}(N)$ matrices $T_{\mathbf{m}}$ of [6]. The corresponding operation to $\mathbf{m} \rightarrow \widehat{\mathbf{m}}$ is matrix transposition, i.e. $T_{\widetilde{\mathbf{m}}}=T_{\mathbf{m}}^{\mathrm{T}}$. Hence we find that, in the matrix picture, the antisymmetric $(N(N-1) / 2)$ $C_{\mathbf{m}}^{-}$matrices form the adjoint and the symmet$\operatorname{ric}(N(N+1) / 2) C_{\mathbf{m}}^{+}$transform as the symmetric rank-two representation of $\mathrm{SO}(N)$. 
Finally we turn to the question of the mass spectrum for membrane states with winding. The mass spectrum of the supermembrane without winding is continuous. This was proven in the $\mathrm{SU}(N)$ regularization [19]. Whether or not nontrivial zero-mass states exist, is not known (for some discussion on these questions, we refer the reader to [7]). Those would coincide with the states of 11-dimensional supergravity. It is often argued that the winding may remove the continuity of the spectrum (see, for instance, [29]). There is no question that winding may increase the energy of the membrane states. A membrane winding around more than one compact dimension gives rise to a nonzero central charge in the supersymmetry algebra. This central charge sets a lower limit on the membrane mass. However, this should not be interpreted as an indication that the spectrum becomes discrete. The possible continuity of the spectrum hinges on two features. First the system should possess continuous valleys of classically degenerate states. Qualitatively one recognizes immediately that this feature is not directly affected by the winding. A classical membrane with winding can still have stringlike configurations of arbitrary length, without increasing its area. Hence the classical instability still persists.

The second feature is supersymmetry. Generically the classical valley structure is lifted by quantum-mechanical corrections, so that the wave function cannot escape to infinity. This phenomenon can be understood on the basis of the uncertainty principle. Because, at large distances, the valleys become increasingly narrow, the wave function will be squeezed more and more which tends to induce an increasing spread in its momentum. This results in an increase of the kinetic energy. Another way to understand this is by noting that the transverse oscillations perpendicular to the valleys give rise to a zero-point energy, which acts as an effective potential barrier that confines the wave function. When the valley configurations are supersymmetric the contributions from the bosonic and the fermionic transverse oscillations cancel each other, so that the wave function will not be confined and can extend arbitrarily far into the valley. This phenomenon indicates that the energy spectrum must be continuous.

Without winding it is clear that the valley configurations are supersymmetric, so that one concludes that the spectrum is continuous. With winding the latter aspect is somewhat more subtle. However, we note that, when the winding density is concentrated in one part of the spacesheet, then valleys can emerge elsewhere corresponding to stringlike configurations with supersymmetry. Hence, as a space-sheet local field theory, supersymmetry can be broken in one region where the winding is concentrated and unbroken in another. In the latter region stringlike configurations can form, which, at least semiclassically, will not be suppressed by quantum corrections.

We must stress that we are describing only the generic features of the spectrum. Our arguments by no means preclude the existence of mass gaps. To prove or disprove the existence of discrete states is extremely difficult. While the contribution of the bosonic part of the Hamiltonian increases by concentrating the winding density on part of the spacesheet, the matrix elements in the fermionic directions will also grow large, making it difficult to estimate the eigenvalues. At this moment the only rigorous result is the BPS bound that follows from the supersymmetry algebra. Obviously, the state of lowest mass for given winding numbers, is always a BPS state, which is invariant under some residual supersymmetry.

\section{REFERENCES}

[1] P.K. Townsend, Phys. Lett. B350 (1995) 184, hep-th/9501068, Phys. Lett. B373 (1996) 68, hep-th/9512062.

[2] E. Witten, Nucl. Phys. B443 (1995) 85, hepth/9503124.

[3] P. Hor̆ava and E. Witten, Nucl. Phys. B460 (1996) 506, hep-th/9510209, Nucl. Phys. B475 (96) 94, hep-th/9603142.

[4] P.K. Townsend, Four Lectures on M-theory, lectures given at the 1996 ICTP Summer School in High Energy Physics and Cosmology, Trieste, hep-th/9612121. 
[5] E.A. Bergshoeff, E. Sezgin and P.K. Townsend, Phys. Lett. B189 (1987) 75.

[6] B. de Wit, J. Hoppe and H. Nicolai, Nucl. Phys. B305 (1988) 545.

[7] B. de Wit and H. Nicolai, in proc. Trieste Conference on Supermembranes and Physics in $2+1$ dimensions, p. 196, eds. M.J. Duff, C.N. Pope and E. Sezgin (World Scient., 1990);

B. de Wit, Supersymmetric Quantum Mechanics, Supermembranes and Dirichlet Particles, Nucl. Phys. B (Proc. Suppl.), 65B (1997) 76, hep-th/9701169.

[8] J. Fröhlich and J. Hoppe, On zero Mass Ground States in Supermembrane Matrix Models, hep-th/9701119.

[9] S. Sethi and M. Stern, D-Brane Bound States Redux, hep-th/9705046;

M. Porrati and A. Rosenberg, Bound states at threshold in supersymmetric quantum mechanics, hep-th/9708119;

[10] E. Witten, Nucl. Phys. B460 (1996) 335, hepth/9510135.

[11] J. Polchinski, S. Chaudhuri and C.V. Johnson, Notes on D-branes, hep-th/9602052;

J. Polchinski, TASI Lectures on D-branes, hep-th/9611050;

C. Bachas, (Half) A Lecture on D-branes, lectures given at the Workshop on Gauge Theories, Applied Supersymmetry and Quantum Gravity, London, 1996, hep-th/9701019.

[12] U. Danielson, G. Ferretti and B. Sundborg, Int. J. Mod. Phys. A11 (1996) 5463, hepth/9603081;

D. Kabat and P. Pouliot, Phys. Rev. Lett. 77 (1996) 1004, hep-th/9603127;

M.R. Douglas, D. Kabat, P. Pouliot and S.H. Shenker, D-branes and short distances in string theory, hep-th/9608024;

J. Polchinski and P. Pouliot, Membrane scattering with M-momentum transfer, hepth/9704029.

[13] O. Aharony and M. Berkooz, Nucl. Phys. B491 (1997) 184, hep-th/9611215;

G. Lifschytz and S.D. Mathur, Supersymmetry and Membrane Interactions in M(atrix) Theory, hep-th/9612087;

G. Lifschytz, Four-brane and six-brane inter- actions in M(atrix) theory, hep-th/9612223;

D. Berenstein and R. Corrado, Phys. Lett. B406 (1997) 37, hep-th/9702108;

V. Balasubramanian and F. Larsen, Relativistic Brane Scattering, hep-th/9703039;

K. Becker and M. Becker, A two-loop test of $M$ (atrix) theory, hep-th/9705091.

K. Becker and M. Becker, J. Polchinski and A. Tseytlin, Higher-order graviton scattering in M(atrix) theory, hep-th/9706072.

[14] T. Banks, W. Fischler, S.H. Shenker and L. Susskind, Phys. Rev. D55 (1997) 5112, hepth/9610043.

[15]L. Susskind, Another conjecture about M(atrix) theory, hep-th/9704080.

[16] J. Polchinski, Phys. Rev. Lett. 75 (1995) 4724, hep-th/9510017.

[17] M.J. Duff, P.S. Howe, T. Inami and K.S. Stelle, Phys. Lett. 191B (1987) 70.

[18] W. Taylor, Phys. Lett. B394 (1997) 283, hepth/9611042;

O. Ganor, S. Rangoolam and W. Taylor, Nucl. Phys. B492 (1997) 191, hepth/9611202;

L. Susskind, T Duality in M(atrix) Theory and S Duality in Field Theory, hepth/9611164.

[19]B. de Wit, M. Lüscher and H. Nicolai, Nucl. Phys. B320 (1989) 135.

[20]B. de Wit, K. Peeters and J.C. Plefka, Phys. Lett. B409 (1997) 117, hep-th/9705225.

[21] A. Strominger, Phys. Lett. B383 (1996) 44, hep-th/9512059;

K. Becker and M. Becker, Nucl. Phys. B472 (1996) 221, hep-th/9602071.

[22] Ph. Brax and J. Mourad, Open supermembranes in eleven dimensions, hepth/9704165, Open supermembranes coupled to $M$ Theory fivebranes, hep-th/9707246.

[23] K. Ezawa, Y. Matsuo and K. Murakami, Matrix regularization of open supermembrane: Towards $M$ Theory five-brane via open supermembrane, hep-th/9707200.

[24] B. de Wit, U. Marquard and H. Nicolai, Commun. Math. Phys. 128 (1990) 39.

[25] K. Ezawa, Y. Matsue and K. Murakami, Lorentz symmetry of supermembrane in light cone gauge formulation, hep-th/9705005. 
[26] J. Goldstone, unpublished; J. Hoppe, in proc. Int. Workshop on Constraint's Theory and Relativistic Dynamics, eds. G. Longhi and L. Lusanna (World Scient., 1987).

[27] N. Kim, and S.-J. Rey, M(atrix) Theory on an Orbifold and Twisted Membrane, hepth/9701139.

[28] T. Banks, N. Seiberg and S.H. Shenker, Nucl. Phys. B490 (1997) 91, hep-th/9612157.

[29] J.G. Russo, Nucl. Phys. B492 (1997) 205, hep-th/9610018;

R. Kallosh, Wrapped Supermembranes, hepth/9612004. 